Vietnam Journal of Mechanics, VAST, Vol. 31, No. 3 \&4 (2009), pp. $293-312$

\title{
SMOOTHED FINITE ELEMENT METHOD FOR TWO-DIMENSIONAL ELASTO-PLASTICITY
}

\author{
Stéphane Pierre Alain Bordascorres ${ }^{1}$, Hung Nguyen-Dang ${ }^{2}$, \\ Quyen Phan-Phuong ${ }^{3}$, Hung Nguyen-Xuan ${ }^{4}$, \\ Sundararajan Natarajan ${ }^{1}$, Marc Duflot ${ }^{5}$ \\ 1 University of Glasgow, G12 8LT, Scotland, UK. \\ ${ }^{2}$ Institut de Mecanique et Genie Civil, Liege, Belgique \\ ${ }^{3}$ University of Technology HCM, Vietnam \\ ${ }^{4}$ University of Natural Sciences - VNU-HCM, Vietnam \\ ${ }^{5}$ Centre of excellence in aeronautical research (CENAERO) \\ Rue des Frères Wright 29 B-6041 Gosselies (Belgium)
}

\begin{abstract}
This communication shows how the smoothed finite element method (SFEM) very recently proposed by G.R. Liu [14] can be extended to elasto-plasticity. The SFEM results are in excellent agreement with the finite element (FEM) and analytical results. For the examples treated, the method is quite insensitive to mesh distortion and volumetric locking. Moreover, the SFEM yields more compliant load-displacement curves compared to the standard, displacement based FE method, as expected from the theoretical developments recently published in 4], 3] and 6].
\end{abstract}

\section{INTRODUCTION}

Recently, G. R. Liu et al. proposed a new finite element method based on strain smoothing, which they coined the Smoothed Finite Element Method - SFEM [14]. Theoretical developments, accuracy, convergence and stability investigations are discussed in 4,6 .

Basically, the strain field is calculated as the spatial average of the compatible strain field. Different numbers of smoothing cells $(n c)$ per element provide the method with different properties.

The SFEM was very recently coupled to the MITC [11, 12 formulation to yield plate [1] and shell [2] elements with particular insensitivity to mesh distortion. The introduction of partition of unity enrichment in the SFEM to create the smoothed extended finite element method (SmXFEM) or Flexible extended finite element method(FleXFEM) was proposed in 6 simplifying integration of discontinuous weak forms. Extensions to polygonal meshes were proposed in [5]. A recent review is given in [6] where these recent results and general properties of the SFEM are discussed in detail. 


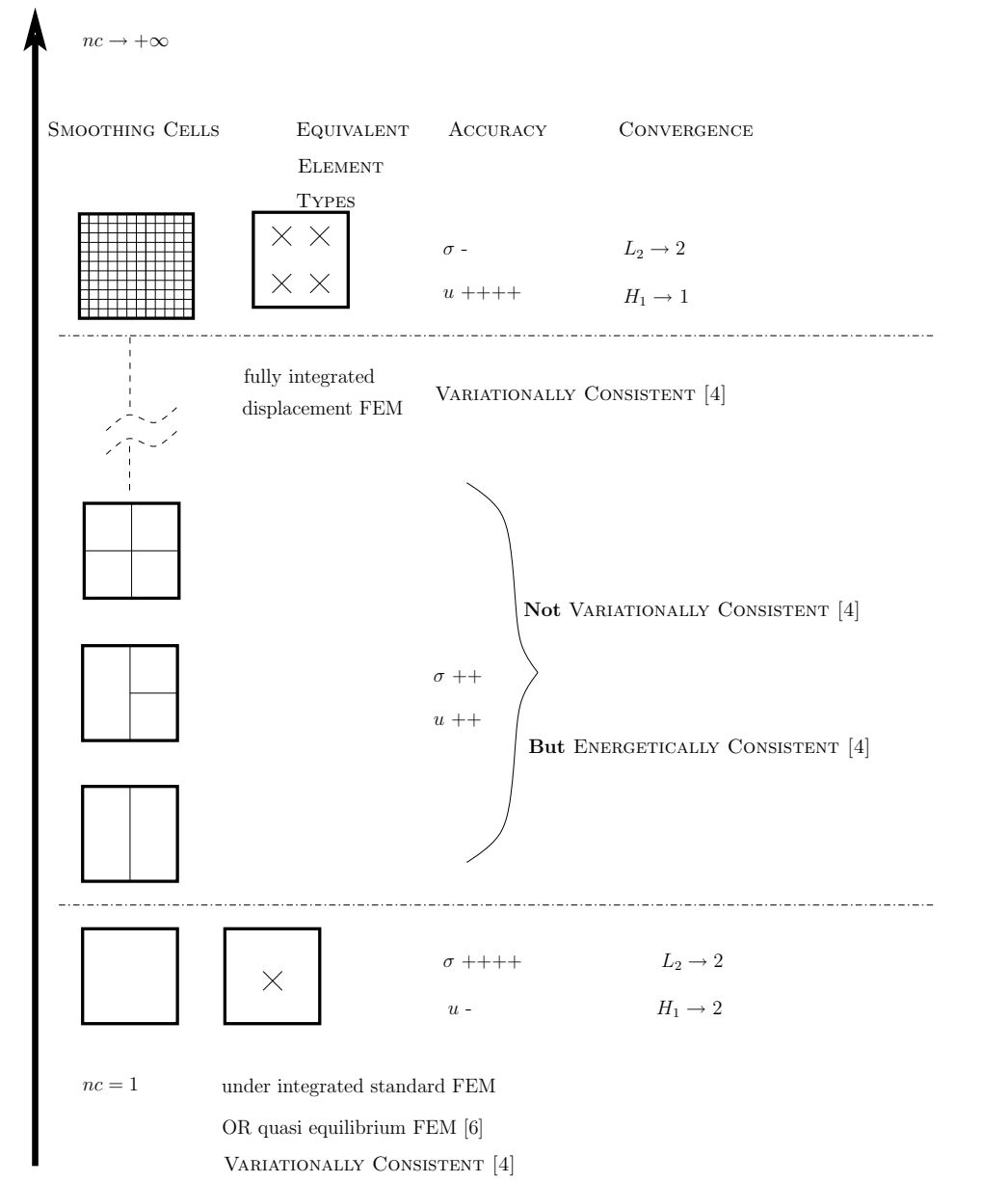

Fig. 1. Summary of properties of the SFEM. The ++ signify greater accuracy, and the -- lesser accuracy. See 6,4 for details.

We recall here the most salient features of the SFEM. A summary of the properties outlined below is given in Figure 1 and Figure 2 .

- Integration can be performed on the boundary of the smoothing cells;

- No isoparametric mapping is necessary (highly distorted meshes are acceptable);

- The computational cost is slightly reduced ( $5 \%$ for four subcells to $20 \%$ for one subcells, in our experience);

- The derivatives of the shape functions are not needed for elliptic problems;

- Low numbers of subcells yield higher stress accuracy, high numbers higher displacement accuracy;

- When the number of subcells tends to infinity, the method becomes equivalent to the displacement-based FEM; 
- The one subcells version usually suppresses locking, it is equivalent to reduced integration and to a quasi equilibrium element formulation and suffers from zero-energy modes due to rank deficiency;

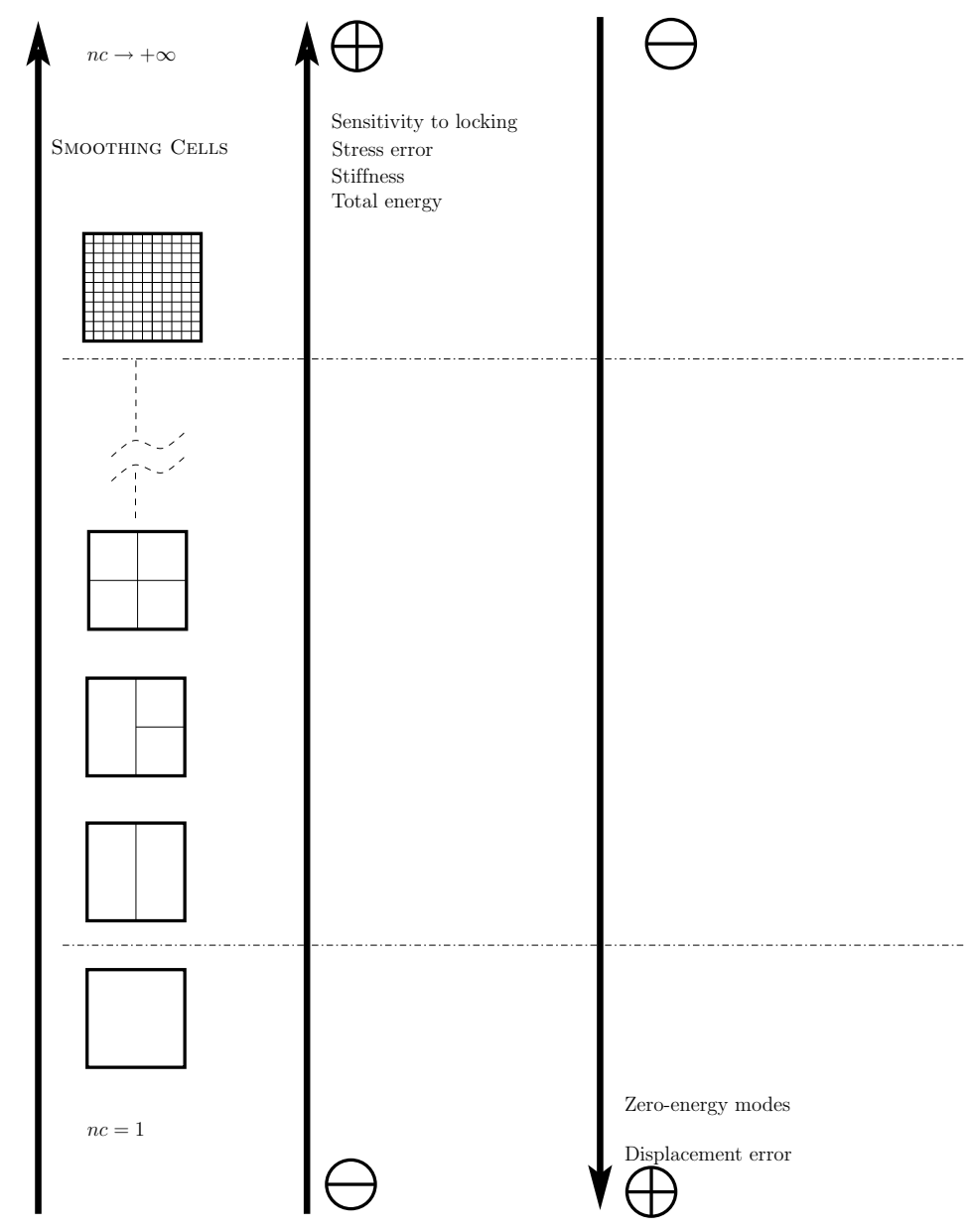

Fig. 2. Scaling of the displacement, stress error, stiffness, stability and total energy depending on the number of subcells. See $[6,4]$ for details.

Figure 3 shows a possible state of stress within a smoothed four-noded quadrilateral. Note that the stresses are discontinuous across the cell walls, but the displacement remains continuous because the shape function of the underlying finite element are used to define the displacement field throughout the element. A typical weight function, used in this paper is shown in Figure 4 . 


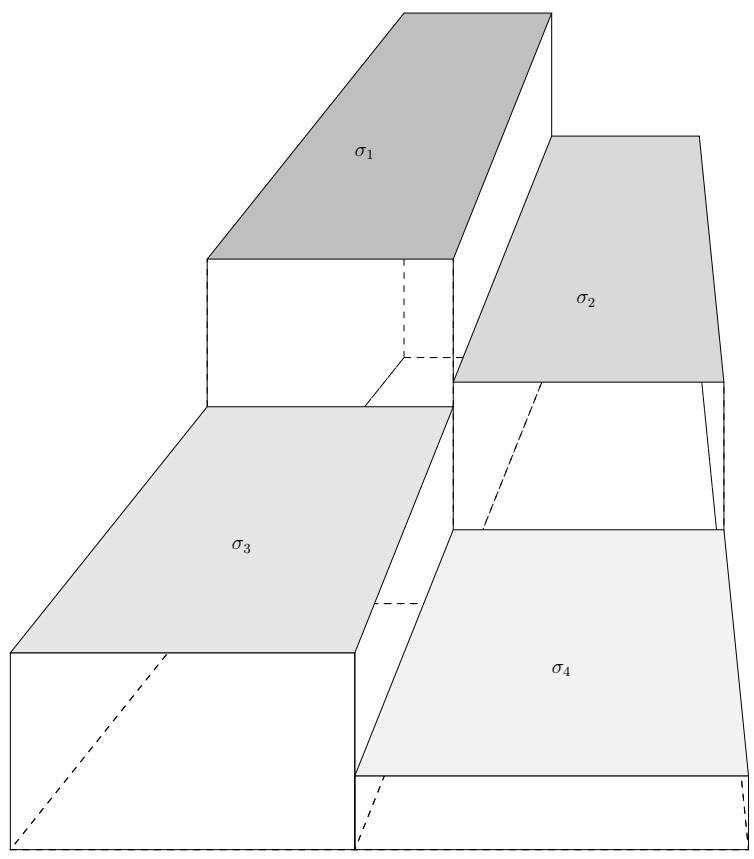

Fig. 3. Stress field in a smoothed finite element. The stress is constant over each smoothing cell, but discontinuous across cells. On the contrary, the displacement field is continuous within the element.

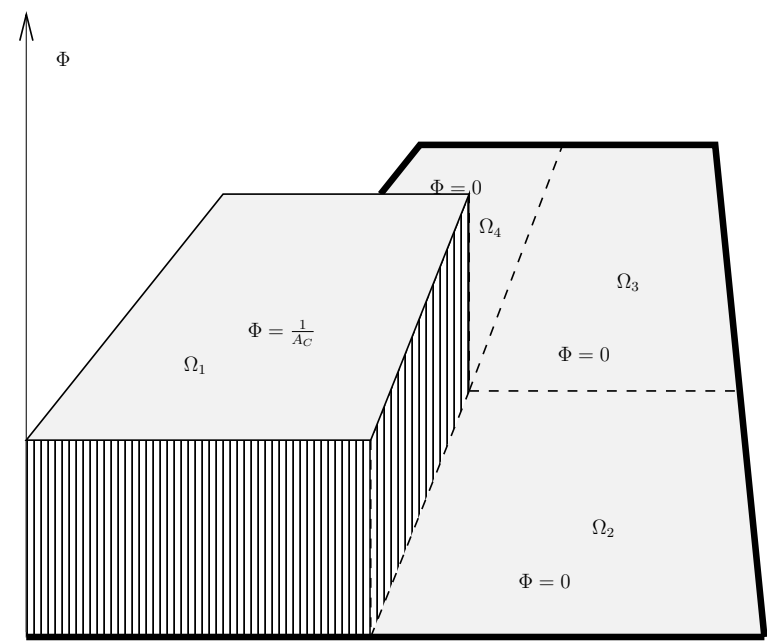

Fig. 4. The weight function is defined, for each subcell as constant equal to the inverse of the area of the subcell and zero elsewhere. This permits transforming the domain integral into a boundary integral over the boundary of the subcell. If a single subcell is used, integration over the boundary of the finite element is recovered. This function has been used in all published work on the SFEM, to date. This figure shows the weight function, $\Phi$ used for subcell $\Omega_{1}$. See Eq. (2). 


\section{SMOOTHED FINITE ELEMENTS}

As described above, the smoothed finite element method (SFEM) was introduced by G. R. Liu et al. [14] in 2006. The full theory is given elsewhere [6, 4], and we do not give details here.

Suffice it to say that the strain field used to write the element stiffness matrix is written as a spatial average of the symmetric gradient of the displacement field, i.e. the compatible strain field. This smoothed strain field writes, at an arbitrary point $\mathbf{x}_{C}$ in an element $\Omega^{h}$

$$
\tilde{\varepsilon}_{i j}^{h}\left(\mathbf{x}_{C}\right)=\int_{\Omega^{h}} \varepsilon_{i j}^{h}(\mathbf{x}) \Phi\left(\mathbf{x}-\mathbf{x}_{C}\right) d \Omega
$$

where $\Phi$ is a smoothing function that generally satisfies the following properties (cf. 30 and Figure 4)

$$
\Phi \geq 0 \text { and } \int_{\Omega^{h}} \Phi d \Omega=1
$$

The strain displacement matrix $\tilde{\mathbf{B}}_{C}$ is constant over each $\Omega_{C}$ and is of the following form

$$
\tilde{\mathbf{B}}_{C}=\left[\begin{array}{llll}
\tilde{\mathbf{B}}_{C 1} & \tilde{\mathbf{B}}_{C 2} & \tilde{\mathbf{B}}_{C 3} & \tilde{\mathbf{B}}_{C 4}
\end{array}\right]
$$

where for all shape functions $i \in\{1, \ldots, 4\}$, the $3 \times 2$ submatrix $\tilde{\mathbf{B}}_{C i}$ represents the contribution to the strain displacement matrix associated with shape function $i$ and cell $C$ and writes (see Figure 5)

$$
\begin{aligned}
\forall i \in\{1,2, \ldots, 4\}, \forall C \in\{1,2, \ldots n c\} \tilde{\mathbf{B}}_{C i} & =\frac{1}{A_{C}} \int_{S_{C}} \mathbf{n}^{T}(\mathbf{x}) N_{i}(\mathbf{x}) d S \\
& =\int_{S_{C}}\left[\begin{array}{cc}
n_{x} & 0 \\
0 & n_{y} \\
n_{y} & n_{x}
\end{array}\right](\mathbf{x}) N_{i}(\mathbf{x}) d S
\end{aligned}
$$

or, since Eq. (??) is computed on the boundary of $\Omega_{C}$ and one Gauss point is sufficient for an exact integration:

$$
\tilde{\mathbf{B}}_{C i}\left(\mathbf{x}_{C}\right)=\frac{1}{A_{C}} \sum_{b=1}^{n b}\left(\begin{array}{cc}
N_{i}\left(\mathbf{x}_{b}^{G}\right) n_{x} & 0 \\
0 & N_{i}\left(\mathbf{x}_{b}^{G}\right) n_{y} \\
N_{i}\left(\mathbf{x}_{b}^{G}\right) n_{y} & N_{i}\left(\mathbf{x}_{b}^{G}\right) n_{x}
\end{array}\right) l_{b}^{C}
$$

where $\mathbf{x}_{b}^{G}$ and $l_{b}^{C}$ are the center point (Gauss point) and the length of $\Gamma_{b}^{C}$, respectively.

The smoothed element stiffness matrix for element $e$ is computed by the sum of the contributions of the subcells(Figure 5 [1

$$
\tilde{\mathbf{K}}^{e}=\sum_{C=1}^{n c} \int_{\Omega_{C}} \tilde{\mathbf{B}}_{C}^{T} \mathbf{D} \tilde{\mathbf{B}}_{C} d \Omega=\sum_{C=1}^{n c} \tilde{\mathbf{B}}_{C}^{T} \mathbf{D} \tilde{\mathbf{B}}_{C} \int_{\Omega_{C}} d \Omega=\sum_{C=1}^{n c} \tilde{\mathbf{B}}_{C}^{T} \mathbf{D} \tilde{\mathbf{B}}_{C} A_{C}
$$

$n c$ is the number of the smoothing cells of the element. Here, the integrands $\tilde{\mathbf{B}}_{C}^{T} \mathbf{D} \tilde{\mathbf{B}}_{C}$ are constant over each $\Omega_{C}$, which permits to take them out of the integral sign.

\footnotetext{
${ }^{1}$ The subcells $\Omega_{C}$ form a partition of the element $\Omega^{h}$ (See Figure 4 .
} 
In the case of a four noded $(i=1,2,3,4)$ quadrilateral element (Q4), the non-local strain displacement submatrix $\tilde{\mathbf{B}}_{C i}$ associated with node $i$ and cell $C$ reads (see Figure 5 )

$$
\tilde{\mathbf{B}}_{C i}=\frac{1}{A_{C}} \int_{\Gamma_{C}}\left(\begin{array}{cc}
N_{i} n_{x} & 0 \\
0 & N_{i} n_{y} \\
N_{i} n_{y} & N_{i} n_{x}
\end{array}\right) d \Gamma=\frac{1}{A_{C}} \int_{\Gamma_{C}} \mathbf{n}^{T} N_{i}(\mathbf{x}) d \Gamma \forall i=1,2,3,4
$$

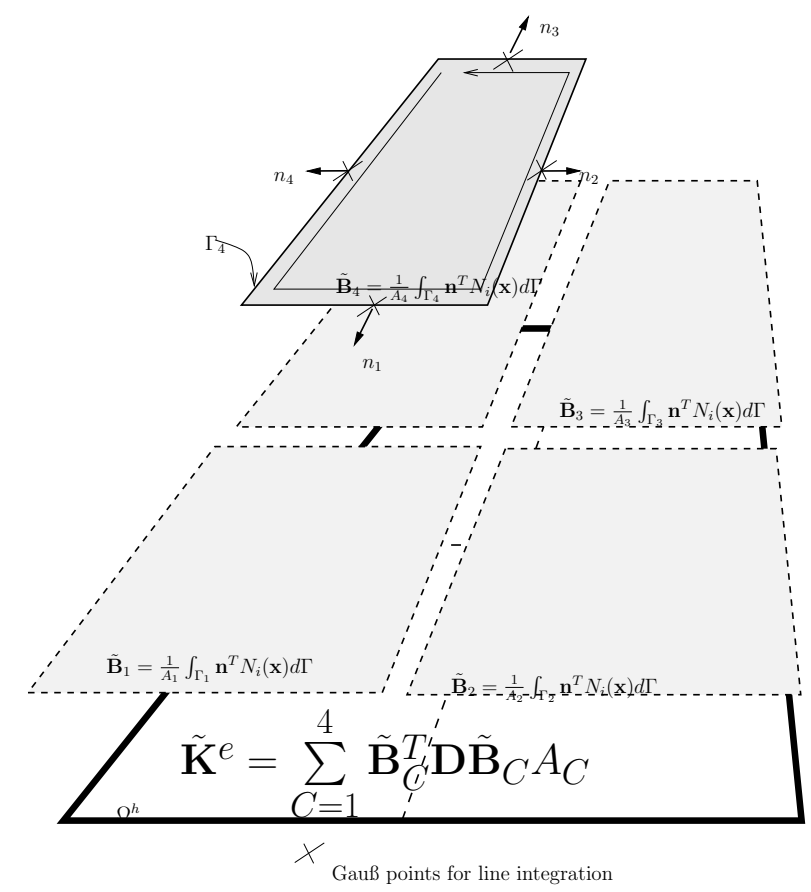

Fig. 5. Schematic representation of the calculation of the smoothed discretised gradient operator on an element $\Omega^{h}$, split into four subcells $\Omega_{C} \cdot\left(A_{i}\right)_{1 \leq i \leq 4}$. In this case one integration point on each edge of the cells is sufficient for exact boundary integration.

From the above, we note the following important points (this assumes a constant smoothing function, $\Phi$ and is used - See Figure 4):

- The stiffness matrix is computed by boundary integration in the physical coordinate system and no isoparametric mapping is required;

- The derivatives of the shape functions do not appear in the expression for the stiffness matrix;

- When a single subcell is employed, the stiffness matrix is calculated by integration along the boundary of the physical element;

- For linear elements, the smoothed stiffness matrix is identical to the standard finite element stiffness (e.g. three-noded linear triangles, two-noded one dimensional elements). 


\section{STABILIZED SMOOTHED FINITE ELEMENT METHOD}

Puso and Solberg 31] proposed a stabilized nodal integration for tetrahedral to address spurious low-energy modes. The proposed method was shown to be stable and optimally convergent for the compressible case. The standard weak form of linear elasticity is given by

$$
a(\mathbf{u}, \mathbf{w})=f(\mathbf{w}), \quad \mathbf{u}, \mathbf{w} \in\left(H^{1}(\Omega)\right)^{3}
$$

for the domain $\Omega \subset \mathbb{R}$. The discrete problem is given by

$$
a_{h}\left(\mathbf{u}_{h}, \mathbf{w}_{h}\right)=f\left(\mathbf{w}_{h}\right)
$$

where $\mathbf{u}_{h}, \mathbf{w}_{h}$ are the discrete trial and test functions, respectively, based on linear tetrahedral interpolation and the discrete bilinear operator $a_{h}\left(\mathbf{u}_{h}, \mathbf{w}_{h}\right)$ is based on average nodal integration. The standard tetrahedral finite element discretization for the equations of motion and applying the preceding notation, the bilinear form can be written as sum over element or a sum over nodes as follows:

$$
a\left(\mathbf{w}_{h}, \mathbf{u}_{h}\right)=\sum_{e=1}^{N_{e}} V^{e} \delta \varepsilon^{e}: \mathbf{C} \varepsilon^{e}
$$

The average nodal gradient $\nabla \mathbf{u}^{I}$ and strain $\varepsilon^{I}$ at node $I$ are defined as

$$
\nabla \mathbf{u}_{h}^{I}=\frac{1}{V^{I}} \sum_{e \in S_{I}} \frac{V^{e}}{4} \nabla \mathbf{u}_{h}^{e}
$$

where $V^{e}=\operatorname{vol}\left(\Omega^{e}\right)$ and $S_{I}$ is the set of all elements common to node $I$ and the average nodal volume is given as

$$
V^{I}=\sum_{e \in S_{I}} \frac{V^{e}}{4}
$$

Puso and Solberg [31] proposed the following nodal strain

$$
a_{h}\left(\mathbf{w}_{h}, \mathbf{u}_{h}\right)=\sum_{I=1}^{N_{n}} V^{I} \delta \varepsilon^{I}: \mathbf{C} \varepsilon^{I}+\sum_{I=1}^{N_{n}} \sum_{e \in S_{I}} \alpha^{e, I} \frac{V^{e}}{4}\left(\delta \varepsilon^{I}-\delta \varepsilon^{e}\right): \tilde{\mathbf{C}}\left(\varepsilon^{I}-\varepsilon^{e}\right)
$$

where $\alpha^{e, I}$ is a stabilization parameter that can potentially depend on element $e$ and node $I$ and $\tilde{\mathbf{C}}$ is the alternate material stiffness. Ideal choice would be $\mathbf{C}=\tilde{\mathbf{C}}$, but for nearly incompressible material, the following choice of $\tilde{\mathbf{C}}$ can be adopted. A uniform value of the stabilization parameter is used, $\alpha^{e, I}=0.05$ ( 31$\left.]\right)$. The form of stabilization stiffness $\tilde{\mathbf{C}}$ is chosen so as to minimize the effects due to volumetric locking while providing the best overall stabilization necessary. For elastic materials, following Lamé parameters $\lambda$ and $\mu$ are used

$$
\tilde{\mu}=\mu, \quad \tilde{\lambda}=\min (\lambda, 25 \tilde{\lambda})
$$

And for plastic materials, a shear modulus based on the plastic tangent modulus $E_{T}$ is used throughout the analysis

$$
\tilde{\mu}=\frac{E_{T}}{2}, \quad \tilde{\lambda}=\min (\lambda, 25 \tilde{\lambda})
$$

For linear hardening, the hardening modulus $H$ is constant and $E_{T}=H$. 


\section{STABILIZED SMOOTHED FINITE ELEMENT METHOD}

In this work, the idea of stabilized nodal integration proposed by Puso and Solberg [31] is extended to smoothed finite element method. The total stiffness $\mathbf{K}$ is given by

$\mathbf{K}_{\text {nodal }}$ and $\mathbf{K}_{\text {elem }}$ is given by

$$
\mathbf{K}=\mathbf{K}_{\text {nodal }}+\mathbf{K}_{\text {elem }}
$$

$$
\begin{array}{r}
\mathbf{K}_{\text {nodal }}=\sum_{c=1}^{n_{c}} \tilde{\mathbf{B}}^{T}(\mathbf{D}-\alpha \tilde{\mathbf{D}}) \tilde{\mathbf{B}} A_{c} \\
\mathbf{K}_{\text {elem }}=\sum_{c=1}^{n_{c}} \alpha A_{c} \tilde{\mathbf{B}}^{T} \tilde{\mathbf{D}} \tilde{\mathbf{B}}
\end{array}
$$

where $\mathbf{D}$ and $\tilde{\mathbf{D}}$ are the stiffness and stabilized stiffness respectively and $\alpha$ is the stabilization parameter, which for the current study is 0.05 .

\section{PLASTICITY IN THE SMOOTHED FINITE ELEMENT METHOD}

The treatment of plasticity in the SFEM is identical in spirit as in the FEM. In this paper only the case of $J_{2}$ plasticity is examined. The return mapping algorithm used in this paper is well-known and not recalled here. The interested reader is referred to the literature for details.

In the SFEM, internal variables are stored in each smoothing cell, which play the same role as the integration points in the FEM. To solve the non-linear problem, the Newton-Raphson algorithm is employed. This algorithm requires two ingredients, at each iteration, $n:^{2}$

The tangent stiffness: With the notations from Section 2 , the tangent stiffness for element $e$ writes

$$
\tilde{\mathbf{K}}_{\text {tangent }}^{e, n+1}=\sum_{C=1}^{n c} \int_{\Omega_{C}} \tilde{\mathbf{B}}_{C}^{T} \mathbf{D}_{C, e p}^{n+1} \tilde{\mathbf{B}}_{C} d \Omega=\sum_{C=1}^{n c} \tilde{\mathbf{B}}_{C}^{T} \mathbf{D}_{C, e p}^{n+1} \tilde{\mathbf{B}}_{C} A_{C}
$$

The residual vector: The residual vector (out of balance forces) is computed as the difference between the external and internal forces. The external forces are known, for each loading step. The internal force vector for element $e$ is computed as follows

$$
\tilde{\mathbf{F}}_{\mathrm{int}}^{e}=\sum_{C=1}^{n c} \int_{\Omega_{C}} \tilde{\mathbf{B}}_{C}^{T} \sigma_{C}^{n+1} d \Omega=\sum_{C=1}^{n c} \tilde{\mathbf{B}}_{C}^{T} \sigma_{C}^{n+1} A_{C}
$$

In the above:

- as in Section 2, the integrands are constant over each $\Omega_{C}$ which allows to take them out of the integral sign and replace the integration by a multiplication by the area $A_{C}$ of the smoothing cell $\Omega_{C}$;

- the smoothed strain displacement matrix $\mathbf{B}_{C}^{T}$ is defined exactly as in Eq. (7);

\footnotetext{
2 as in the derivation of Section 2 the "tilde" denotes "smoothed" quantities.
} 
- $v \operatorname{sigma} a_{C}^{n+1}$ is the state of stress in cell $C$ obtained from the radial return algorithm;

- $\mathbf{D}_{C, e p}^{n+1}$ is the elasto-plastic tangent operator in cell $\Omega_{C}$ for the current iteration.

The last two quantities in the list above are independent of the discretization scheme, and calculated exactly as in the standard FEM.

\section{NUMERICAL EXAMPLES}

\subsection{Notation}

The smoothed FEM four-noded elements are noted SCkQ4, where $k$ stands for the number of subcells. For instance, the SC4Q4 is the four-noded finite element with four smoothing cells. In this paper, the von Mises yield criterion is used. The implementation uses a radial return algorithm. The interval variables are stored at the smoothing cell level, which play the same role as the integration points in the FEM.

\subsection{General remarks}

In the numerical results below, it will be seen that the SFEM behaves better than the FEM for distorted meshes. For the latter, convergence of the Newton-Raphson algorithm is not attained for medium to high levels of mesh distortion. Strain smoothing alleviates this problem.

In general, convergence is attained within a maximum of 7-10 iterations for a precision of 1e-14 on the Euclidian norm of the residual. The number of iterations to convergence increases in the vicinity of the failure load.

\subsection{The footing problem}

\subsubsection{Problem and geometry}

This first example is concerned with the calculation of the bearing capacity of a superficial footing. The geometry and material properties are given in Figure 6. The load $q$ on the footing is increased until failure occurs.

\subsubsection{Theoretical failure load}

Terzaghi 7 gives the following expression, based on a Mohr-Coulomb yield surface, for the ultimate load on a strip superficial foundation:

$$
q_{u}=c N_{c}+\gamma t N_{q}+\frac{1}{2} \gamma b N_{\gamma}
$$

where $c$ is the cohesion, $\gamma$ the unit weight of the soil, $t$ the foundation depth, $b$ the foundation width and $N_{c}, N_{q}, N_{\gamma}$ are coefficients which are function of the friction angle of the soil $\phi$.

Using a von Mises criterion under plane strain conditions, an appropriate size adjustment must be done in order to achieve the same ultimate load as with a Mohr-Coulomb criterion 8, 9]:

$$
c=\frac{\sqrt{3}}{2} k \quad \varphi=0
$$




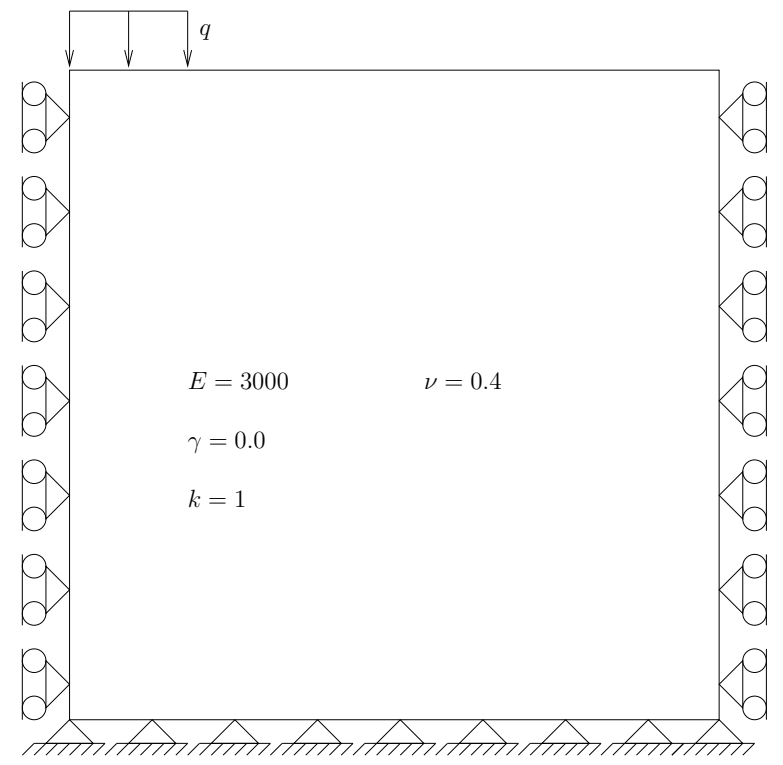

Fig. 6. Geometry of the footing problem. $\gamma$ is the specific weight of the soil, $k=\sigma_{y} / \sqrt{3}$, where $\sigma_{y}$ is the yield stress.

Here, $t=0, \varphi=0, \phi=0$ leads to $N_{c}=5.711$, which finally yeilds:

$$
q_{u}=c N_{c}=5 \mathrm{kN} / \mathrm{m}
$$

\subsubsection{Numerical results}

Convergence of the Newton-Raphson algorithm. The Newton-Raphson algorithm converges within 5-7 iterations for a tolerance of 1e-014. The solution fails to converge for an applied load of $6 \mathrm{kN} / \mathrm{m}$, which is satisfactory for such a crude mesh.

Failure mechanism and plastic zone. Figure 7 illustrates the failure mechanism and shows the plastic domain when the failure load is attained. These results are qualitatively very similar to those presented in 9 .

Effect of the number of subcells on the load-displacement curve. Figure 8 shows the load displacement curve (applied load $q$ versus displacement at the center of the footing). From this figure, it can be inferred that the load displacement curve is practically insensitive to the number of subcells used in the simulation. More interestingly, it is observed that the SFEM solution is always more compliant (the slope of the load-displacement curve is smaller) than the FEM solution, which is in agreement with the theoretical results presented in [6, 4].

From Figure 8, we note that the vertical displacement at node 125 is approximately $-0.85 \mathrm{e}-03 \mathrm{~m}$ for an applied load $q=6 \mathrm{kN} / \mathrm{m}$ for all element types. In [9], the vertical displacement at the soil/footing interface is found to be $-0.9 \mathrm{e}-03 \mathrm{~m}$ for $q=7 \mathrm{kN} / \mathrm{m}$. 


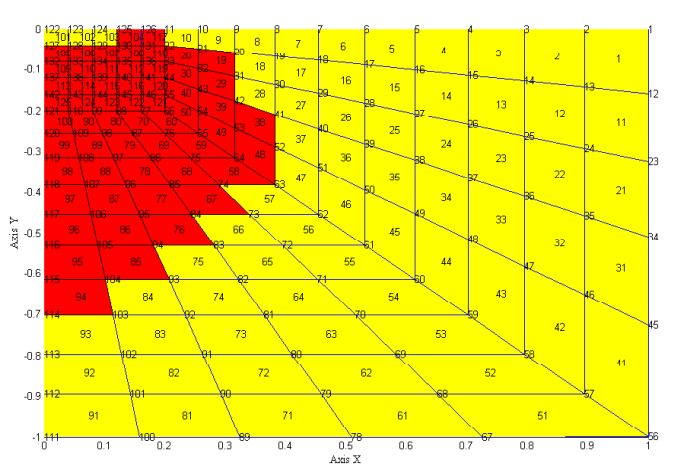

(a) Plastic domain

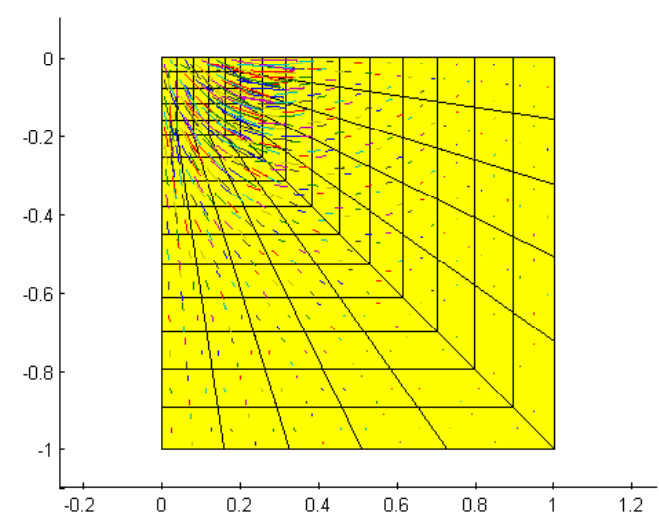

(b) Failure mechanism

Fig. 7. Plastic domain and failure mechanism $(p=6 \mathrm{kN} / \mathrm{m})$

Convergence of the load-displacement curve with mesh refinement. To further test the method, load displacement curves ${ }^{3}$ for a series of mesh densities (4 smoothing cells are used) are shown in Figure 9, smaller load increments were used in the vicinity of the failure load. The figure shows the convergence of the load displacement curve with mesh refinement.

Influence of mesh distortion on the load-displacement curve. The effectiveness of the method is studied for a series of meshes comprising severely distorted element, including quadrilaterals with obtuse angles. Irregular meshes are generated as explained in [4] and the coordinates have the following general form:

$$
\begin{aligned}
x^{\prime} & =x+\Delta x \cdot r_{c} \cdot \alpha_{i r} \\
y^{\prime} & =y+\Delta y \cdot r_{c} \cdot \alpha_{i r}
\end{aligned}
$$

where $\Delta x$ and $\Delta y$ are initial regular element sizes in the $x$ - and $y$ - directions, respectively; $r_{c}$ is a computer generated random number between -1.0 and 1.0 and $\alpha_{i r}$ is a prescribed irregularity factor whose value is chosen between 0.0 and 0.5. The bigger the value of $\alpha_{i r}$, the more irregular the shape of the generated elements. Figure 10 shows two sample meshes used for the present study. Figure 11 shows the load displacement curves ${ }^{4}$ for various irregularity factors $\alpha_{i r}$ ranging from 0 (regular mesh) to 0.4 (very irregular). Four smoothing cells are used and smaller load increments empliyed in the vicinity of the failure load. The figure shows that the results for the distorted meshes, with irregularity parameter $\alpha_{i r}=0.1,0.2$ are comparable to that of the regular mesh $\left(\alpha_{i r}=0.0\right)$ until the applied load reaches $q=7 \mathrm{kN} / \mathrm{m}$, beyond which some small oscillations are observed, which may be attributed to the distortion. The amplitude of these oscillations is however not significant. A smiliar trend is observed for the distorted mesh with an irregularity parameter as high as $\alpha_{i r}=0.4$. It can be concluded that the results are not highly sensitive to mesh distortion.

\footnotetext{
3 the right-most under the distributed load is used and perfect plasticity is assumed $(H=0)$ $\left.\frac{2 E}{3}\right)$.

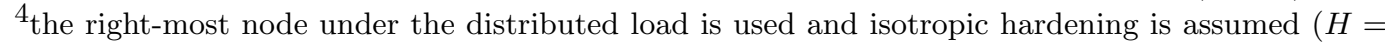




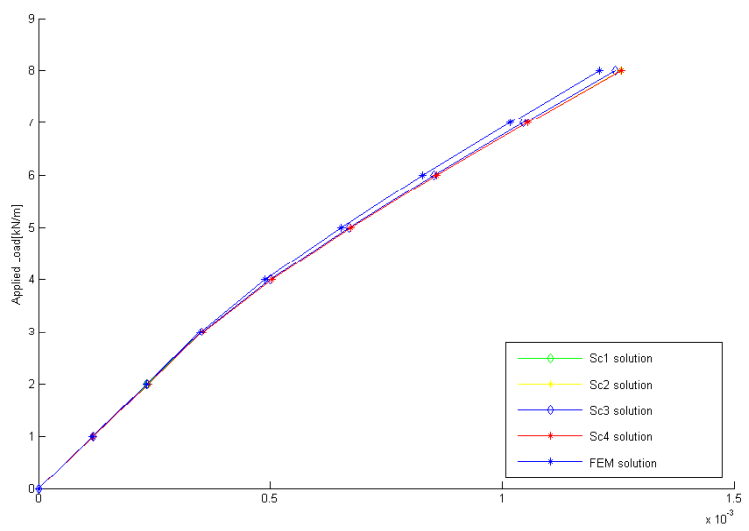

Fig. 8. Load displacement curve (vertical displacement measured at node 125)

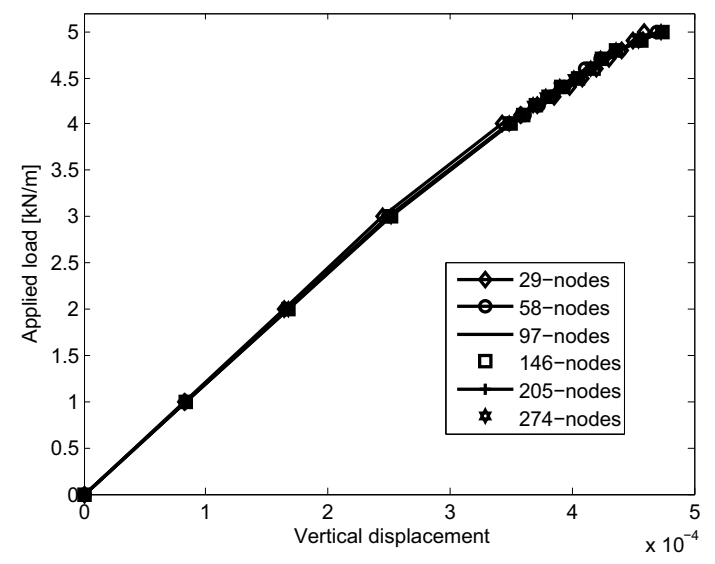

Fig. 9. Load displacement curve (vertical displacement measured at node situated at the right-most of the distributed load section; 4 smoothing cells are used in each finite element. )

Using the standard FEM, the Newton-Raphson algorithm is divergent for $\alpha_{i r}=0.3$, while in the case of the SFEM, convergence is attained up to extremely distorted meshes $\left(\alpha_{i r}=0.4\right)$. A comparison of the load displacement curves obtained using the FEM and the SFEM for several levels of mesh distortion are shown in Figure 13.

\subsection{Hollow cylinder under internal pressure}

In this example, a thick cylinder under pressure is considered. The analytical solution is due to Hill can be found in [10]. The setup for this problem is given in Figure 14.

The internal and external radius of the cylinder are $b=0.1$ and $a=0.2 \mathrm{~m}$. The radius of the plastic zone is denoted by $c$. Young's modulus $E=21000 \mathrm{kN} / \mathrm{m}^{2}$ and Poisson's ratio $\nu=0.3$ and plane strain conditions are assumed. The von Mises criterion is used with a yield stress $\sigma_{y}=24 \mathrm{kN} / \mathrm{m}^{2}$ which corresponds to $k=\frac{\sigma_{y}}{3}=13.8564 \mathrm{kN} / \mathrm{m}^{2}$. The internal 


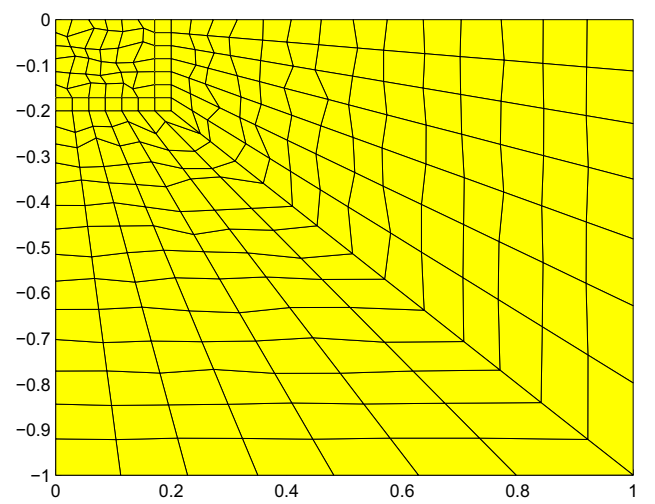

(a) Distorted mesh $\alpha_{i r}=0.2$

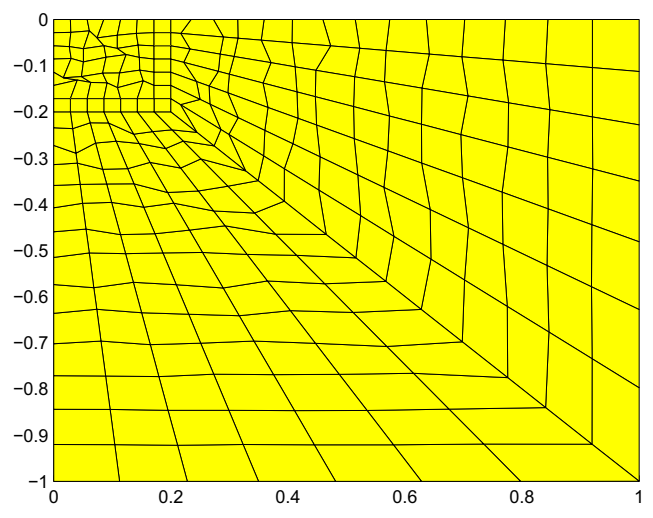

(b) Distorted mesh $\alpha_{i r}=0.4$

Fig. 10. Domain discretization with irregular elements. Note that some elements have obtuse angles.

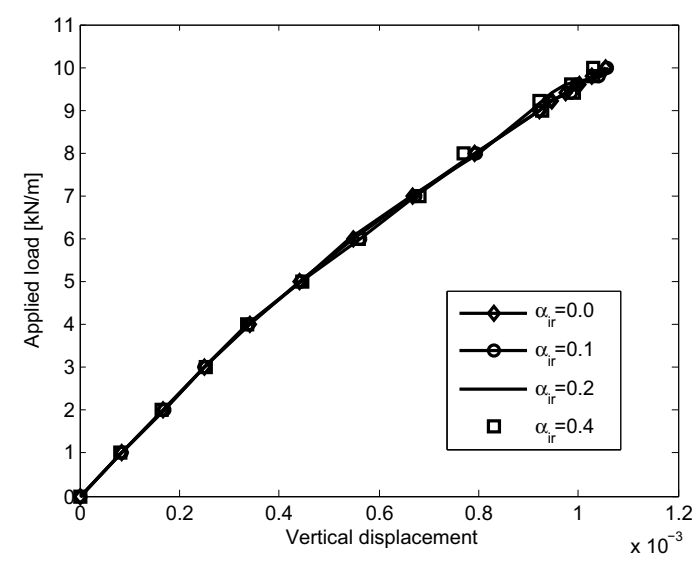

Fig. 11. Load displacement curve for different levels of mesh distortion (vertical displacement measured at the node situated at the right-most of the distributed load section; 4 smoothing cells are used in each finite element. Isotropic hardening is assumed.)

pressure $p$ is varied between $8 \mathrm{kN} / \mathrm{m}^{2}$ and $18 \mathrm{kN} / \mathrm{m}^{2}$, this value corresponding to the total plastification of the cylinder and its failure. Only one-quarter of the cylinder is modeled because of symmetry.

First, the stresses $\sigma_{r}$ and $\sigma_{\theta}$ are calculated, for six cases: a) $p=8 \mathrm{kN} / \mathrm{m}^{2}$, b) $p=$ $14 \mathrm{kN} / \mathrm{m}^{2}$, c) $18 \mathrm{kN} / \mathrm{m}^{2}$ both for perfectly plastic material $(H=0)$ and for a hardening material, where $H=\frac{2 E}{3}=14000 \mathrm{kN} / \mathrm{m}^{2}$. The results are shown in Figure 15 . The plastic zones are depicted in Figure 16. 


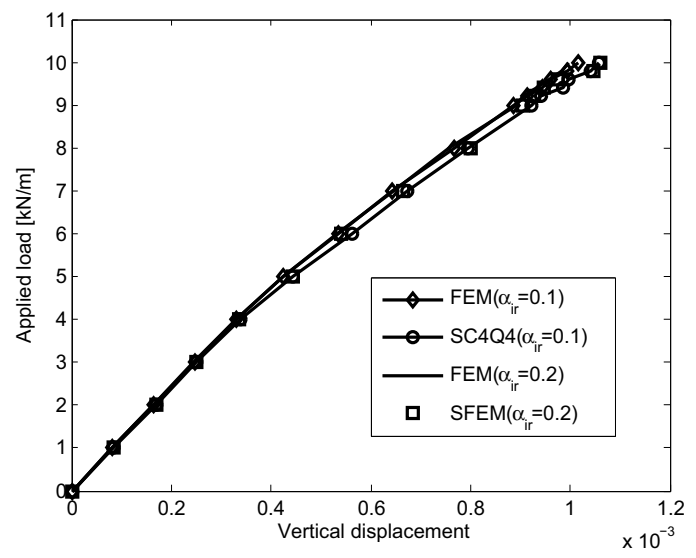

Fig. 12. Comparison with FEM. Load displacement curve for different levels of mesh distortion (vertical displacement measured at the node situated at the rightmost of the distributed load section; 4 smoothing cells are used in each finite element. Isotropic hardening is assumed.)

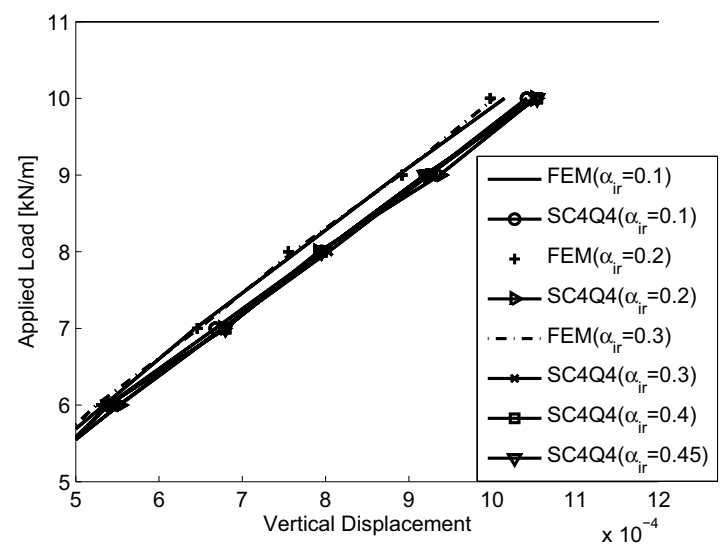

Fig. 13. Comparison with FEM. Load displacement curve for different levels of mesh distortion (vertical displacement measured at the node situated at the rightmost of the distributed load section; 4 smoothing cells are used in each finite element. Isotropic hardening is assumed.)

The evolution of the radius of the plastic zone, $c$, is presented in Figure 16 for both the perfectly plastic case and the hardening case. As expected, hardening requires a larger load to produce the same plastic zone size as in the perfectly plastic case. The results obtained are in good agreement with the theoretical solutions given in [10].

Comparing the SFEM stress solution to that of FEM, Figure 17 shows that for moderately distorted meshes, the FEM solution is already oscillatory, while the SFEM retains high accuracy. 


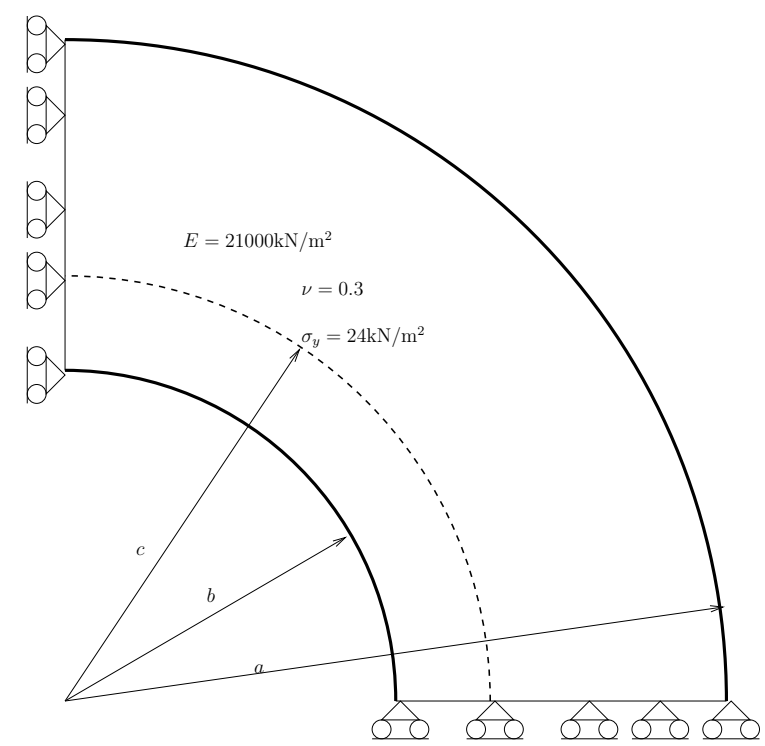

Fig. 14. Geometry for the thick cylinder problem.
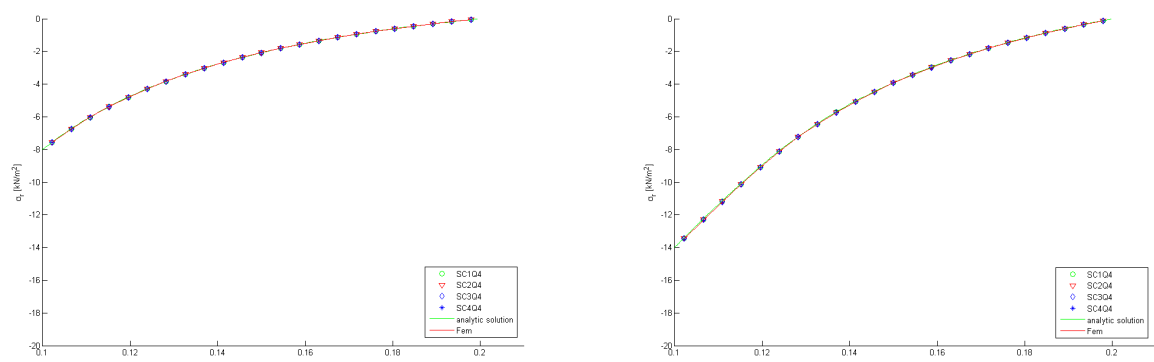

(a) The radial stress for $p=8 \mathrm{kN} / \mathrm{m}^{2}$ with (b) The radial stress for $p=14 \mathrm{kN} / \mathrm{m}^{2}$ with $\nu=0.3, H=0$ $\nu=0.3, H=0$
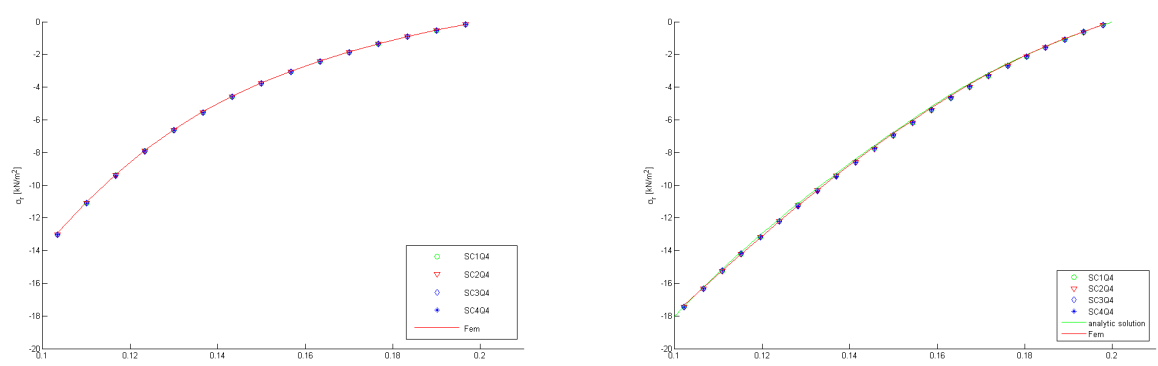

(c) The radial stress for $p=14 \mathrm{kN} / \mathrm{m}^{2}$ with (d) The radial stress for $p=18 \mathrm{kN} / \mathrm{m}^{2}$ with $\nu=0.3, H=2 / 3 E$ $\nu=0.3, H=0$ 


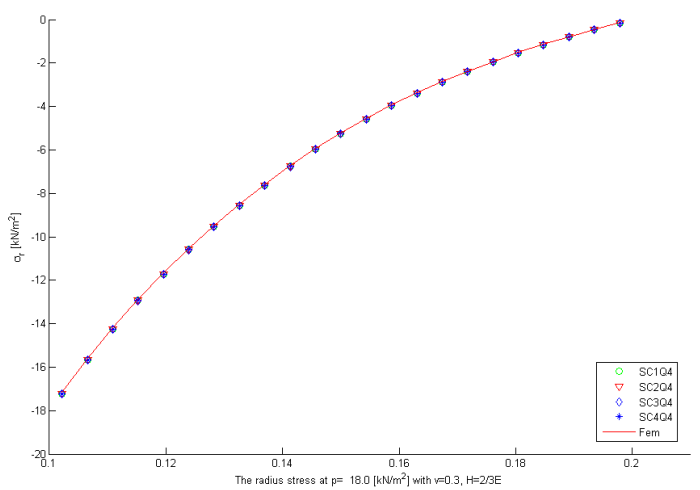

(e) The radial stress for $p=18 \mathrm{kN} / \mathrm{m}^{2}$ with $\nu=0.3, H=$ $2 / 3 E$

Fig. 15. Radial stress for various load levels.

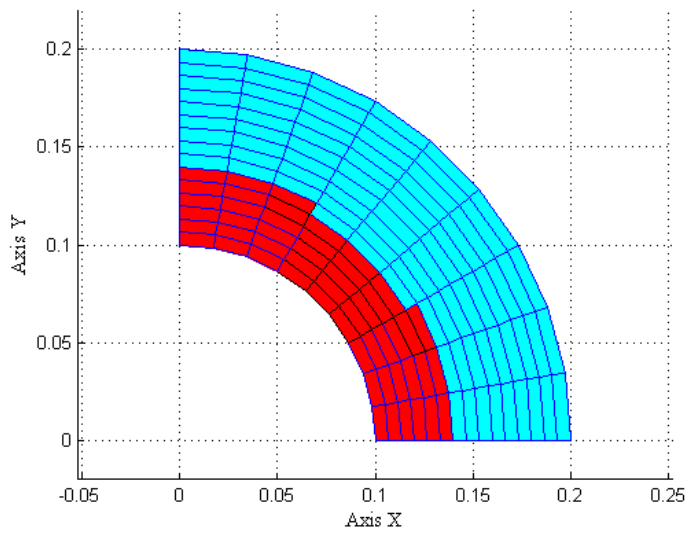

(a) $p=16 \mathrm{kN} / \mathrm{m}^{2} c=0.1333 \mathrm{~m}$ in the case $H=0$

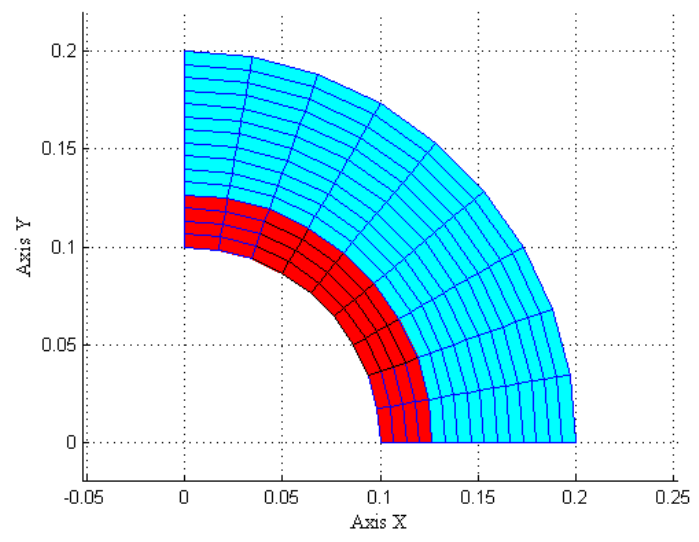

(b) $p=16 \mathrm{kN} / \mathrm{m}^{2} c=0.1266$ in the case $H=2 / 3 E$

Fig. 16. Mesh and plastic domain at $p=16 \mathrm{kN} / \mathrm{m}^{2}$ with and without hardening

We now consider the same problem for a nearly incompressible material $(\nu=$ 0.49999) in plane strain conditions. As shown in Figure 18 and Figure 19, the stresses $\sigma_{r}$ and $\sigma_{\theta}$ are very accurate for all the SC1Q4, SC2Q4, SC3Q4, SC4Q4 element. 


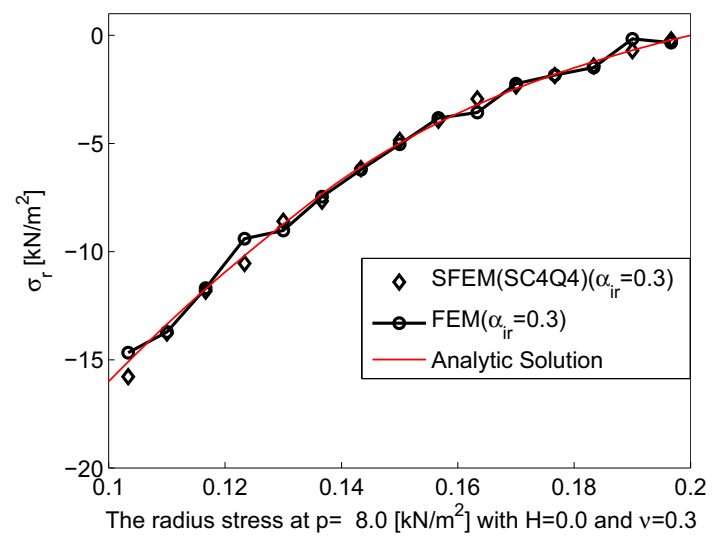

Fig. 17. Thick cylinder problem, comparison of SFEM with FEM for moderately distorted mesh $\left(\alpha_{i r}=0.3\right.$. The figure shows the radial stress for no hardening and $\nu=0.3$. Note the absence of spurious oscillations in the SFEM solution (4 subcells were used here).
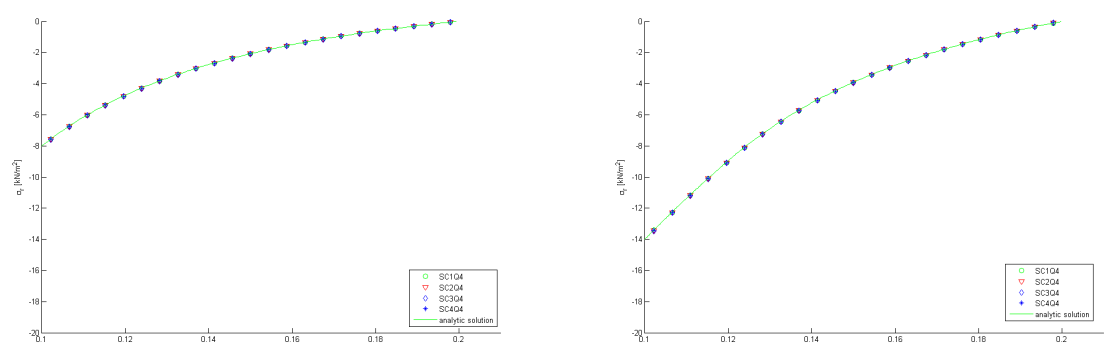

(a) The radial stress for $p=8 \mathrm{kN} / \mathrm{m}^{2}$ with (b) The radial stress for $p=14 \mathrm{kN} / \mathrm{m}^{2}$ with $\nu=0.49999, H=0$ $\nu=0.49999, H=0$
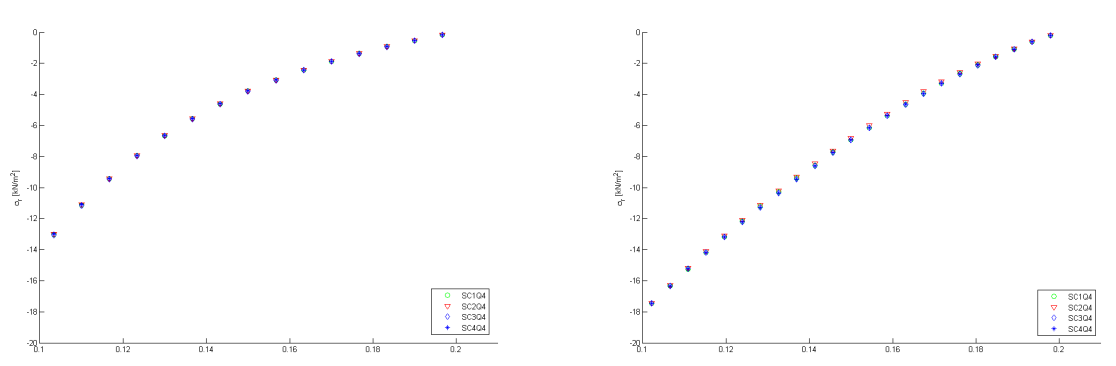

(c) The radial stress for $p=14 \mathrm{kN} / \mathrm{m}^{2}$ with (d) The radial stress for $p=18 \mathrm{kN} / \mathrm{m}^{2}$ with $\nu=0.49999, H=2 / 3 E$ $\nu=0.49999, H=0$ 


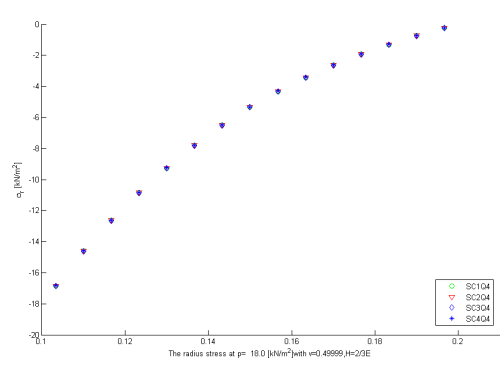

(e) The radial stress for $p=18 \mathrm{kN} / \mathrm{m}^{2}$ with $\nu=0.49999, H=2 / 3 E$

Fig. 18. Radial stress for different load levels, perfect and hardening plasticity in the incompressible case.
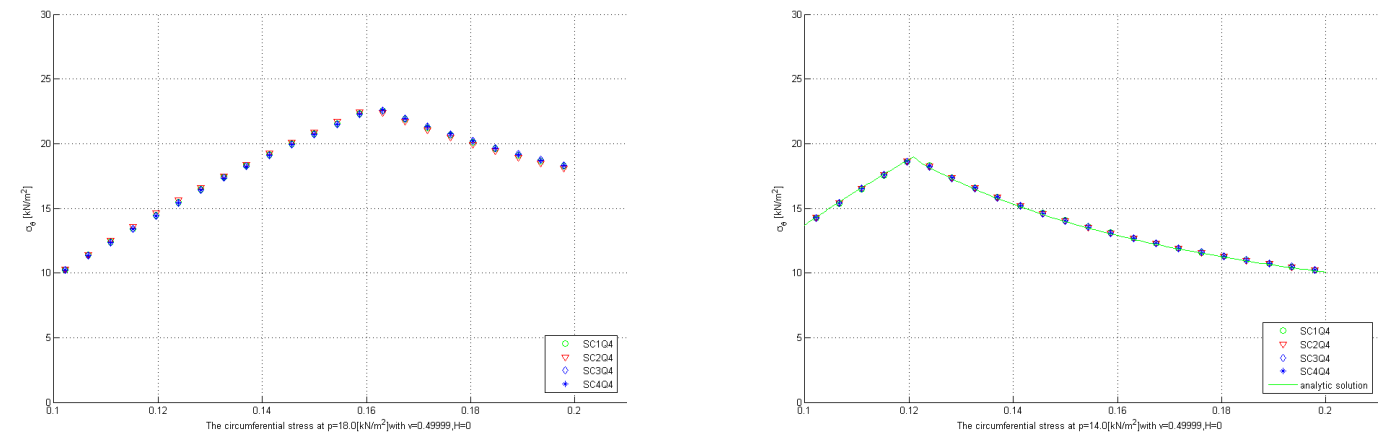

(a) The hoop stress for $p=18 \mathrm{kN} / \mathrm{m}^{2}$ with (b) The hoop stress for $p=14 \mathrm{kN} / \mathrm{m}^{2}$ with $\nu=0.49999, H=0 \quad \nu=0.49999, H=0$

Fig. 19. Hoop stress for different load levels, perfect and hardening plasticity in the incompressible case.

\section{CONCLUSION}

This paper presented the extension of recent work on the smoothed finite element (SFEM) to elasto-plasticity. The four elements tested, all based on smoothed strain fournoded quadrilateral elements produced load-displacement and stress results which compare very well with the analytical and finite element solution.

In particular, the smoothed finite element method was shown to produce accurate results, even for extremely distorted meshes including elements with obtuse angles. The method was also shown to be insensitive to volumetric locking.

This work is the first step toward the longer term goal of simulating large elastoplastic deformation while limiting the amount of remeshing for which the aptitude of the method in dealing with highly distorted meshes is promising. 


\section{REFERENCES}

[1] H. Nguyen-Xuan, S. Bordas, and H. Nguyen-Dang. A smoothed finite element method for plate analysis. Computer Methods in Applied Mechanics and Engineering, in press, doi:10.1016/j.cma.2007.10.008, 197(13-16) 1184-1203, 2007.

[2] H. Nguyen-Xuan, S. Bordas, and H. Nguyen-Dang. A smoothed finite element method for shell analysis. Computer Methods in Applied Mechanics and Engineering, in press, 2008.

[3] H. Nguyen-Xuan, Stéphane Bordas and H. Nguyen-Dang Smooth finite elements: convergence, accuracy and properties International Journal for Numerical Methods in Engineering, in press doi:10.1002/nme.2146, 2007

[4] G. R. Liu, T. T. Nguyen, K. Y. Dai, and K. Y. Lam. Theoretical aspects of the smoothed finite element method (SFEM). International Journal for Numerical Methods in Engineering, 71(8):902-930, 2007.

[5] K.Y. Dai, G. R. Liu, and T. T. Nguyen. An n-sided polygonal smoothed finite element method (nSFEM) for solid mechanics. Finite Elements in Analysis and Design, 43(11):847-860, 2007.

[6] Stéphane Bordas, Timon Rabczuk, Nguyen-Xuan Hung, Sundararajan Natarajan, Tino Bog, Quan Do Minh and Hiep Nguyen Vinh. Advances and recent developments in smoothed finite element method (SFEM) and first results in the smoothed extended finite element method (SmFEM). Computers and Structures in press, 2008.

[7] K. Terzaghi, Mécanique théorique des sols, Dunod, Paris, 1951

[8] Z-SOIL user's manual, 1998

[9] Stéphane Commend, Thomas Zimmermann, Object-oriented nonlinear finite element programming, Comp. Methods Appl. Mech. Engrg. 139 (1996)

[10] R. Hill, The mathematical theory of plasticity (Oxford University Press, 1950)

[11] K.J. Bathe and E.N. Dvorkin. A four-node plate bending element based on Mindlin/Reissener plate theory and a mixed interpolation. International Journal for Numerical Methods in Engineering, 21:367 - 383, 1985.

[12] K.J. Bathe and E.N. Dvorkin. A formulation of general shell elements. the use of mixed interpolation of tensorial components. International Journal for Numerical Methods in Engineering, 22:697 - 722, 1986.

[13] J.C. Simo and T.J.R. Hughes, On the variational foundation of assumed strain methods, ASME Journal of Applied Mechanics, 53:51 54, 1986

[14] G.R. Liu, K.Y. Dai, and T.T. Nguyen, A smoothed finite element for mechanics problems, Computational Mechanics, DOI 10.1007/s00466-006-0075-4, 2006.

[15] T. Belytschko and S.P. Xiao, Stability analysis of particle methods with corrected derivatives, Compt. Math. Appl. 43 (2002)

[16] J. Dolbow and T.Belytschko, Numerical integration of the Galerkin weak form in meshfree methods, Compt. Mech. 23 (1999)

[17] T.Rabczuk and T.Belytschko, Cracking particles: a simplified meshfree method for arbitrary evolving cracks, Internal.J.Numer.Methods Engrg. 61(2004) 1359.

[18] Liu G.R. (2003), Meshfree methods - Moving beyond the Finite Element Method. CRC Press.

[19] J.J. Monaghan, An introduction to SPH, Comput. Phys. Commun. 48(1988)

[20] Bui-Quoc Tinh, Application of the element free Galerkin method for dual analysis, EMMC, Master thesis(2005)

[21] G.R. Johnson, S.R. Beissel, Normalized smoothing functions for SPH impact computations, Int. J. Number. Methods Engrg. 39(1996)

[22] P. Randles and L. Libersky, Smoothed particle hydrodynamics: Some recent improvements and applications, Comput. Methods Appl. Mech. Engrg .146(1968) 
[23] S.P. Xiao, T. Belytchsko, Material stability analysis of particle methods Advances in Com. Mathematics (Springer 2005) 23

[24] P.W. Randles, L.D. Libersky, Normalized SPH with stress point, Int. J. Numer. Methods Engrg.48 (2000)

[25] Szeidl, G.: Boundary integral equations for plane problems in terms of stress functions of order one, Journal of Computational and Applied Mechanics, 2(2), (2001), 237-261.

[26] S. Beissel, T. Belytschko, Nodal integration on the element free Galerkin method, Comput. Methods App, Mech. Engrg. 139 (1996).

[27] L. Cueto-Felgueroso, I. Colominas, G. Mosqueira, F. Navarrina and M. Casteleiro, On the Galerkin formulation of the smoothed particle hydrodynamics method, Int. J. Number. Meth. Engng 2004.

[28] J.W. Swegle, D.L. Hooks and S.W. Attaway, Smoothed particle hydrodynamics stability analysis, J. Comput. Phys. 116 (1995).

[29] J.S. Chen, C.T. Wu, S. Yoon, and Y. You. A stabilized conforming nodal integration for Galerkin mesh-free methods. International Journal for Numerical Methods in Engineering, 50:435-466, 2001.

[30] J.W. Yoo, B. Moran, and J.S. Chen. Stabilized conforming nodal integration in the naturalelement method. International Journal for Numerical Methods in Engineering, 60:861-890, 2004.

[31] M.A. Puso and J. Solberg, A stabilized nodally integrated tetrahedral. International Journal for Numerical Methods in Engineering, 67:841-867, 2006.

Received September 30, 2009

\section{PHƯƠNG PHÁP PHẦN TỬ HỬU HẠN TRƠN CHO ĐÀN-DẺO HAI CHIỀU}

Báo cáo này chỉ ra làm thể nào để mở rộng phương pháp phần tử hữu hạn trơn (SFEM) được G.R. Liu đề nghị trong thời gian gần đây cho bài toán đàn-dẻo. Các kết quả của SFEM cực kỳ phù hợp với các kết quả của phương pháp phần tử hữu hạn và giải tích. Với các ví dụ khảo sát, SFEM ít nhạy cảm với sự vặn của lưới và nghẽn thể tích. Thêm vào đó, như mong đợi từ sự phát triển lý thuyết được công bố gần đây trong [1], [3] và [6], SFEM đưa tới các biểu đồ lực-chuyển vị thích hợp hơn so với phương pháp phần tử hữu hạn chuẩn dựa trên trường chuyển vị. 